\title{
PUMA- and Bax-induced autophagy contributes to apoptosis
}

\author{
KS Yee ${ }^{1}$, S Wilkinson ${ }^{1}$, J James ${ }^{2}$, KM Ryan ${ }^{1}$ and KH Vousden ${ }^{\star, 1}$
}

The p53-inducible BH3-only protein PUMA is a key mediator of p53-dependent apoptosis, and PUMA has been shown to function by activating Bax and mitochondrial outer membrane permeabilization. In this study, we describe an ability of PUMA to induce autophagy that leads to the selective removal of mitochondria. This function of PUMA depends on Bax/Bak and can be reproduced by overexpression of Bax. The induction of autophagy coincides with cytochrome $c$ release, and taken together the results suggest that PUMA functions through Bax to induce mitochondrial autophagy in response to mitochondrial perturbations. Surprisingly, inhibition of PUMA or Bax-induced autophagy dampens the apoptotic response, suggesting that under some circumstances the selective targeting of mitochondria for autophagy can enhance apoptosis.

Cell Death and Differentiation (2009) 16, 1135-1145; doi:10.1038/cdd.2009.28; published online 20 March 2009

Apoptotic cell death plays an important role in normal development and the deregulation of apoptotic pathways can contribute to the genesis of various diseases, including cancer. The activation of apoptosis by oncogenic stress signals is thought to represent a key tumor suppressive mechanism that prevents the outgrowth of nascent tumor cells. ${ }^{1}$ Accordingly, the inhibition or loss of at least some aspects of the apoptotic response pathways is a frequent characteristic of cancers in humans and model systems. One of the key pathways through which apoptosis is regulated depends on the control of mitochondrial outer membrane permeability (MOMP), which in turn regulates the release of apoptogenic factors from the mitochondria that can trigger a cascade of caspase activation. ${ }^{2}$ MOMP can be influenced by a family of proteins that consists of both pro- and antiapoptotic members and show some similarity to the first of these proteins to be described, Bcl2. ${ }^{3}$ At the core of the pathway lie Bax and Bak - two proapoptotic family members that function directly at the mitochondria to regulate MOMP. ${ }^{4} \mathrm{Bcl} 2, \mathrm{Bcl}-\mathrm{xL}$ and other antiapoptotic family members act to inhibit Bax and Bak, and a large number of so-called BH3-only proteins function to promote apoptosis either by inhibiting $\mathrm{Bcl} / \mathrm{Bcl}-\mathrm{xL}$ or by activating Bax and Bak. ${ }^{5}$ Of particular interest when considering a role of apoptosis in the suppression of cancer development is a BH3-only protein named PUMA. ${ }^{6}$ Expression of PUMA is regulated at the transcriptional level by $p 53$, a tumor suppressor protein that is either directly or indirectly inactivated in most cancers. p53 functions to prevent tumor development through a number of different mechanisms, including the ability to reduce and repair genotoxic damage, and through an important contribution to the activation of oncogene-induced senescence. ${ }^{7}$ p53 can also activate apoptosis in several cell types, and in some tissues the induction of p53-dependent apoptosis is critical for tumor suppression. ${ }^{8}$ Although p53 can activate the expression of a number of $\mathrm{BH} 3$-only proteins, studies in genetically modified mice have suggested that PUMA is the central mediator of apoptotic response of p53 in most cell types. ${ }^{6}$

Autophagy refers to the highly regulated and evolutionarily conserved process of turnover and maintenance of cellular components that is required for cellular homeostasis. ${ }^{9}$ It is a multistep process where portions of cytoplasm and organelles are engulfed in double-membrane vesicles called autophagososomes. These structures then fuse with lysosomes, resulting in the destruction of their contents by the acid hydrolases provided by the lysosome. Although autophagy is generally considered nonspecific, in certain instances there appears to be selection for specific organelles such as ribosomes, the endoplasmic reticulum, peroxisomes or mitochondria. The selective loss of mitochondria has been termed mitophagy. ${ }^{10}$ Although autophagy occurs constitutively in most cells, it can also be induced under conditions of stress such as starvation, hypoxia or drug treatment. ${ }^{11}$ In response to nutrient deprivation, autophagy clearly functions to promote survival - at least in the short term - by providing a source of energy through catabolism of the cell's own components. ${ }^{12}$ Mitophagy has also been shown to contribute to survival by targeting the removal of damaged mitochondria and so eliminating the source of apoptogenic signals or reducing ROS levels. ${ }^{13,14}$ However, despite these contributions to cell survival under stress (usually associated with tumor promotion), components of the autophagic pathways have been shown to function as tumor suppressors, suggesting that the ability to carry out autophagy somehow protects

\footnotetext{
${ }^{1}$ The Beatson Institute for Cancer Research, Garscube Estate, Switchback Road, Bearsden, Glasgow G61 1BD, UK and ${ }^{2} \mathrm{CHIPS}$, College of Life Sciences, Dundee University, Dow Street, Dundee DD1 5EH, UK

${ }^{*}$ Corresponding author: KH Vousden, Tumour Suppressor Laboratory, The Beatson Institute for Cancer Research, Garscube Estate, Switchback Road, Bearsden, Glasgow G61 1BD, UK. Tel: + 440141330 2424; Fax: + 440141943 0372; E-mail: k.vousden @ beatson.gla.ac.uk

Keywords: PUMA; Bax; autophagy

Abbreviations: MOMP, mitochondrial outer membrane potential; BH3, bcl2 homology 3; ROS, reactive oxygen species; z-VAD-fmk, benzyloxycarbonyl-Val-AlaAsp(OMe)-fluoromethylketone; LC3, (microtubule-associated protein 1) light chain 3; MEF, mouse embryo fibroblast; ER, endoplasmic reticulum; MPT, mitochondria permeability transiition

Received 01.9.08; revised 26.1.09; accepted 19.2.09; Edited by G Melino; published online 20.3.09
} 
from tumor development. ${ }^{11,15}$ This is further supported by the general observation that many oncogenes inhibit autophagy while tumor suppressor proteins can activate autophagy. ${ }^{16}$ This may reflect a role for autophagy in maintaining genome stability or could reflect a recently described function for autophagy in supporting apoptosis in some systems. ${ }^{17,18}$

A number of recent studies have revealed interesting crosstalk between the apoptotic and autophagic pathways, with the identification of several proteins that can play a role in both responses. ${ }^{19} \mathrm{Bcl} 2$ and $\mathrm{Bcl}-\mathrm{xL}$, for example, have been shown to inhibit autophagy as well as apoptosis by binding to Beclin-1, an autophagy-inducing protein that contains a $\mathrm{BH} 3$ domain. ${ }^{20}$ Interestingly, proapoptotic $\mathrm{BH} 3$-only proteins such as BNIP3 and Bad, as well as pharmacological BH3 mimetics, can function to induce autophagy by competitively disrupting the interaction between Beclin-1 and Bcl2 or Bcl-xL. 14,21 Conversely, Atg5 - a protein required for the formation of autophagosomes - can be rendered proapoptotic following cleavage by calpain. In this case, the Atg5 cleavage product acquires the ability to move to the mitochondria, interact with $\mathrm{Bcl}-\mathrm{xL}$ and drive cytochrome $c$ release and caspase activation. $^{22}$ In light of these increasing links between the two responses, we investigated whether the p53-inducible proapoptotic protein PUMA might also have a function in regulating autophagy.

\section{Results}

Our earlier studies have shown that PUMA can localize to the mitochondria through a C-terminal hydrophobic domain, and by virtue of the $\mathrm{BH} 3$ domain-dependent interaction with Bcl2. ${ }^{23}$ While studying the subcellular localization of Flagtagged PUMA, we noted that the expression of PUMA reproducibly resulted in a characteristic clustering of the mitochondria around the nucleus. Co-staining of cells for Tom20, a mitochondrial outer membrane protein, revealed a filamentous network of mitochondria in untransfected cells, whereas cells expressing PUMA (which colocalizes with Tom 20) showed an aggregate clustering of the mitochondria around the nucleus - an effect that was seen within $12 \mathrm{~h}$ of transfection of PUMA into a number of different cell lines (Figure 1a and $b$ and Supplementary Figure 1A). In time course experiments, we noted that the mitochondrial clustering was observed as soon as PUMA expression could be detected, indicating that the changes in mitochondria occurs concurrently with or very rapidly after PUMA localization. In a number of cells, at later time points, the initial clustering of mitochondria in PUMA-expressing cells appeared to be followed by a loss of mitochondria. Identical results were seen after staining for different mitochondrial proteins such as COX I and COX IV (data not shown). To confirm that this mitochondria loss was due to PUMA expression, we coexpressed GFP-C1 with PUMA to give an independent marker of which cells had been transfected with PUMA. This approach showed clearly that by $24 \mathrm{~h}$ post-transfection, the depletion of mitochondria was evident only in the GFPpositive cells (Figure 1c and Supplementary Figure 1B). Our earlier studies have shown that expression of extremely low levels of PUMA (below the level of detection by western blot) were sufficient to induce apoptosis, and we found that the clustering and loss of mitochondria were also induced by similarly low levels of PUMA expression (data not shown). Although the mitochondrial clustering and loss could be seen most clearly when the experiments were carried out in the presence of the pan-caspase inhibitor z-VAD-fmk to inhibit the loss of cells through PUMA-induced apoptosis, these effects of PUMA expression were also observed in the absence of z-VAD-fmk (Figure 1d), indicating that the loss was not a consequence of caspase inhibition. Although the activation of apoptosis in the absence of z-VAD-fmk made it difficult to quantify how many PUMA-expressing cells showed mitochondrial loss, at earlier time points $(8 \mathrm{~h})$ it was clear that the mitochondrial clustering occurred at a similar rate in the presence or absence of z-VAD-fmk (Supplementary Figure 1C).

It is well established that bulk degradation of cytoplasmic components is usually due to autophagy. Recent studies have illustrated the role of $\mathrm{BH} 3$ domain proteins in the regulation of autophagy, as well as apoptosis. We therefore considered the possibility that PUMA - in common with some other BH3-only proteins - may be stimulating autophagy and the consequent degradation of organelles including the mitochondria. A common method for detecting autophagy is by monitoring the conversion of GFP-tagged microtubule-associated protein 1 light chain 3 (MAP-LC3), from a diffuse cytosolic form (LC3-I) to a lipidated form (LC3-II) that forms part of the autophagic membrane and can be visualized as puncta accumulation by fluorescence microscopy. ${ }^{24}$ Using GFPtagged LC3 to monitor the formation of autophagosomes, we found that PUMA strongly induced the formation of GFP-LC3 puncta, along with mitochondrial clustering (Figure 2a and Supplementary Figure 2A). An examination of the localization of the LC3 puncta showed that there was a generally closer localization with mitochondria (stained by Tom20) than with other organelles such as the golgi (stained with GM130) (Figure 2b). Although there was clearly not complete concurrence of the LC3 and Tom20 staining, the degree of overlap was similar to that seen earlier under conditions where selective autophagic degradation of mitochondria - or mitophagy - had been identified. ${ }^{21}$ We therefore looked more directly at the PUMA-induced loss of various organellespecific proteins by western blotting (Figure 2c), which showed that while PUMA expression clearly led to the loss of mitochondrial proteins (COXII and COXIV), there was no change in the levels of proteins marking other organelles, including Golgi (GM130) and ER (Calnexin). In agreement with these observations, PUMA-expressing cells showed no reduction in ER or golgi staining by immunofluorescence (data not shown). These results therefore suggested that PUMA expression leads to an increased rate of autophagy that results in the selective loss of mitochondria.

A number of recent reports have shown that $\mathrm{BH} 3$ domain proteins, such as BNIP3 and Bad, can induce autophagy by releasing Beclin-1 - an essential component of the autophagic pathway - from an inhibitory interaction with $\mathrm{Bcl} 2$ or $\mathrm{Bcl}-\mathrm{xL}$. This function of BNIP3 would depend on its $\mathrm{BH} 3$ domain for interaction with $\mathrm{Bcl} 2 / \mathrm{BclXL}$, but would not be expected to require other mediators of the apoptotic response such as Bax or Bak. To test whether PUMA may be functioning in a similar manner, we first examined the contribution of the PUMA BH3 
a
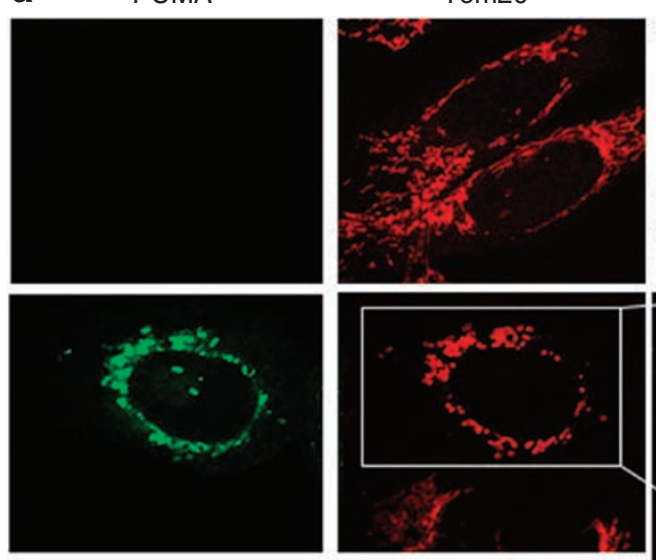
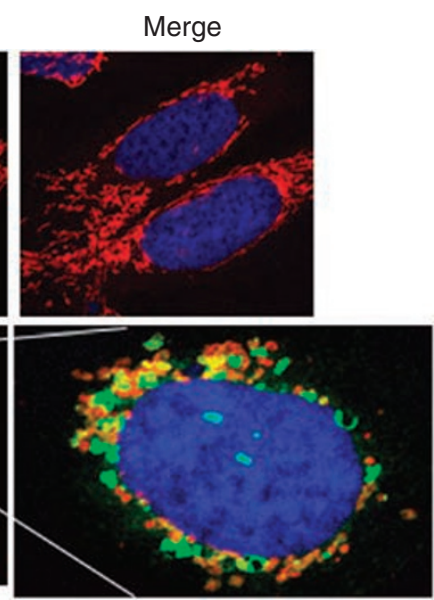

b

PUMA Tom20
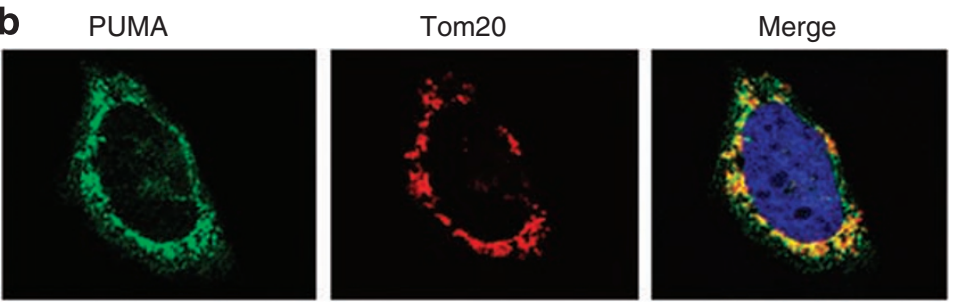

C

GFP

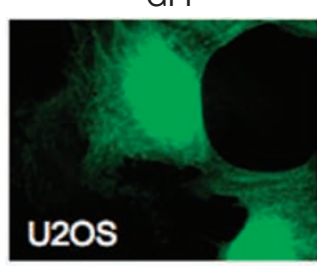

Tom20
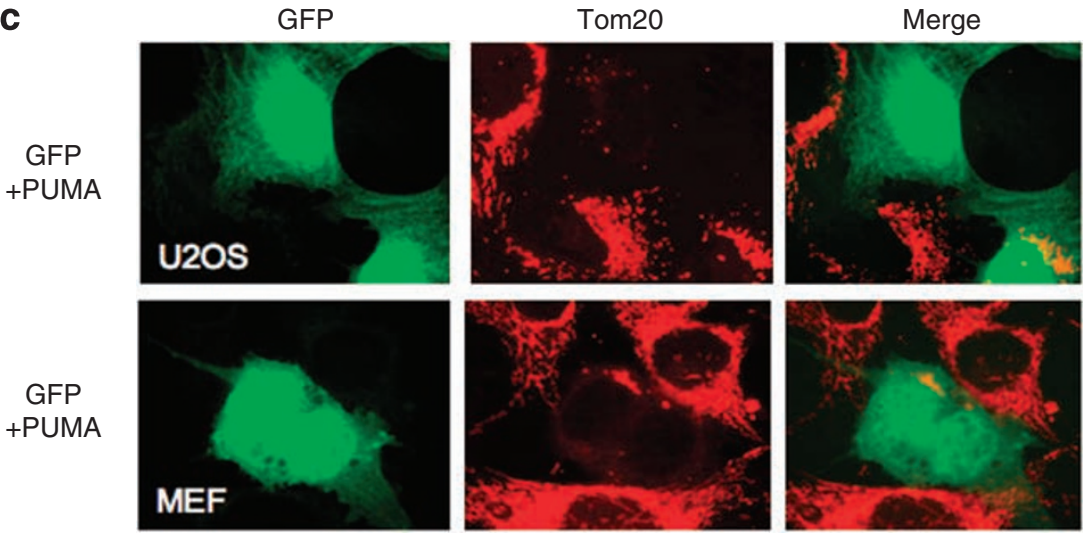

d

PUMA
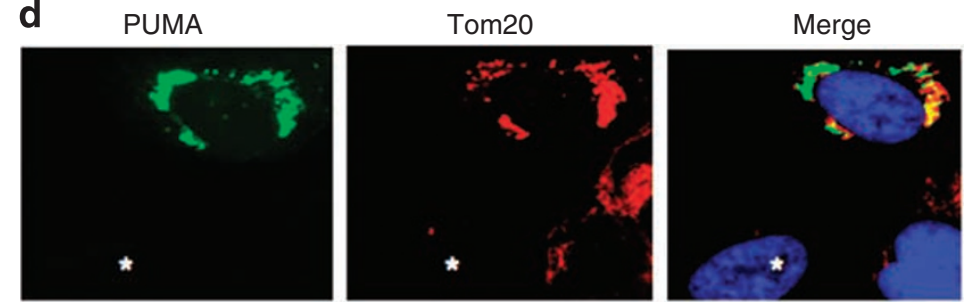

Figure 1 PUMA induces perinuclear accumulation and loss of mitochondria in different cell types. (a) U2OS cells were transfected with GFP-tagged PUMA expression plasmid and maintained in the presence of z-VAD-fmk to inhibit apoptosis. After $24 \mathrm{~h}$, cells were fixed and subject to immunofluorescence analysis. Mitochondria were stained using anti-Tom20 antibody. The top panels show normal mitochondrial morphology in control transfected U2OS cells. (b) Saos-2 TetOn PUMA cells were treated with doxycycline to induce PUMA. After $24 \mathrm{~h}$, PUMA was detected using an anti-Flag antibody while mitochondria were stained as above. (c) U2OS or MEF cells were cotransfected with a combination of pEGFP plasmid and PUMA-expression plasmid and maintained in z-VAD-fmk. After $24 \mathrm{~h}$, cells were fixed and immunostained with antiTom20 antibody. GFP positivity indicated cells that co-expressed PUMA and showed loss of mitochondria (detected with anti-Tom20). (d) U2OS cells were transfected with GFP-PUMA and maintained without z-VAD-fmk. After $12 \mathrm{~h}$, mitochondrial clustering was evident in GFP-PUMA-positive cells, with some cells (an example is indicated by *) showing loss of the mitochondrial marker

domain. Deletion of three amino acids within the $\mathrm{BH}$ domain resulted in a failure of PUMA to induce the mitochondrial clustering seen following expression of the wild-type protein
(Figure 3a) and this mutant was also defective in the induction of LC3 puncta (Figure 3b). Interestingly, a PUMA mutant lacking the $\mathrm{C}$-terminal domain - but retaining the $\mathrm{BH} 3$ domain 
a
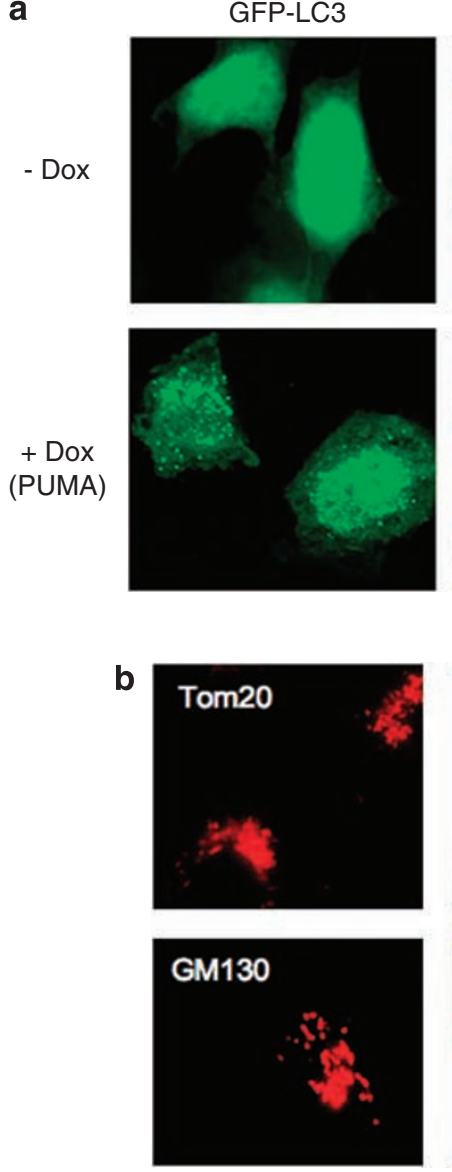
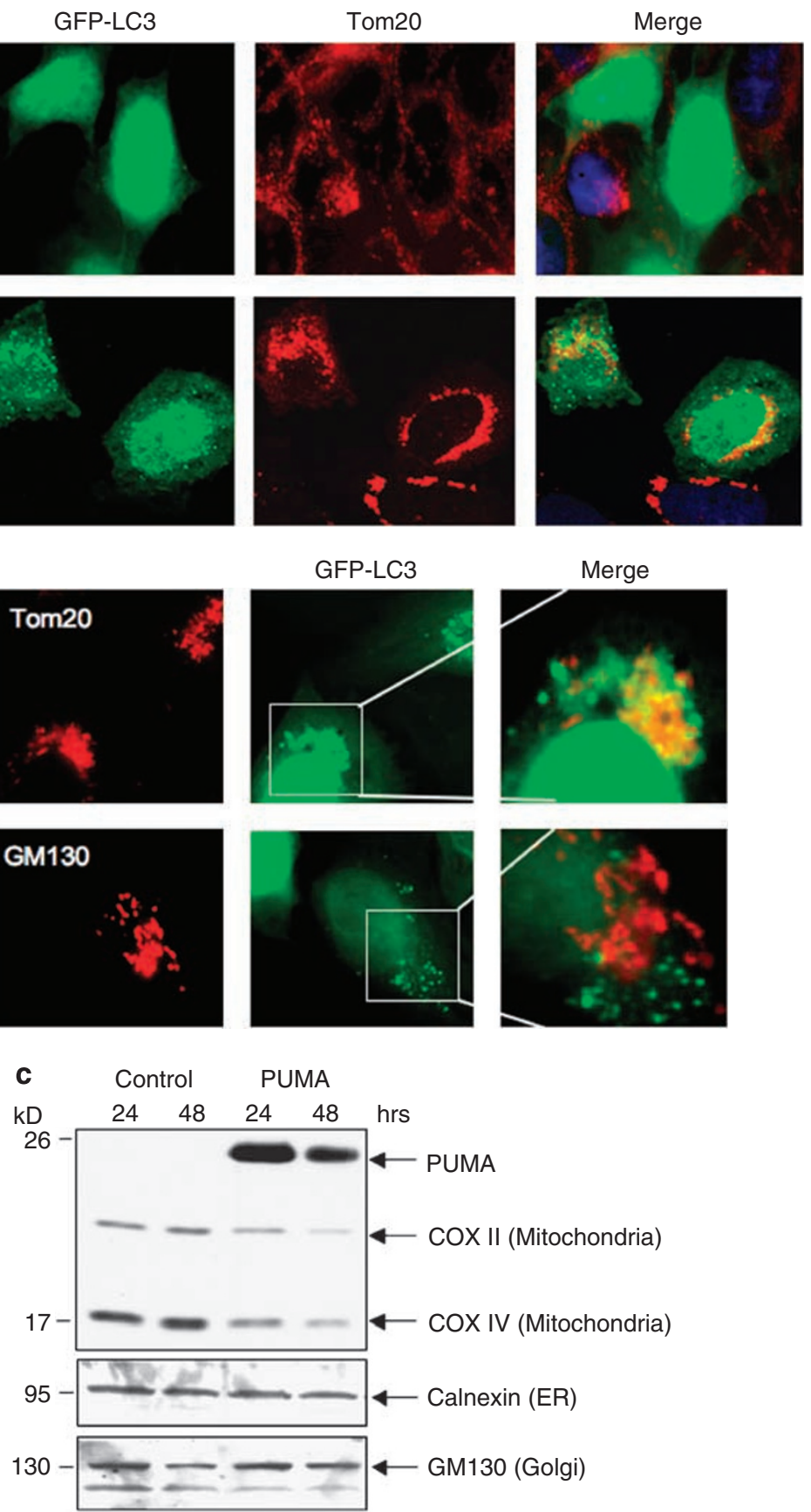

Figure 2 PUMA induces accumulation of GFP-LC3 puncta, which preferentially colocalize with mitochondria. (a) Saos-2 TetOn PUMA cells were infected with GFP-LC3expressing adenovirus, left for $16 \mathrm{~h}$ and then treated with doxycycline. Cells were maintained in the presence of $\mathrm{z}$-VAD-fmk throughout the experiment. After $24 \mathrm{~h}$, cells were fixed and immunostained with anti-Tom20 antibody to visualize the mitochondria. (b) U2OS cells were infected with GFP-LC3-expressing adenovirus, left for $16 \mathrm{~h}$ and then transfected with PUMA-expression plasmid. Cells were maintained in the presence of $\mathrm{Z}$-VAD-fmk throughout the experiment. After $24 \mathrm{~h}$, cells were fixed and immunostained with anti-Tom20 (upper panel) or anti-GM130 (lower panel). Cells exhibiting GFP-LC3 puncta were then observed under a fluorescence microscope to compare the colocalization of GFP-LC3 puncta with mitochondria (Tom20) or Golgi (GM130). (c) U2OS cells were transfected with vector control or PUMA-expression plasmid and maintained in the presence of Z-VAD-fmk throughout the experiment. Cells were harvested 24 or $48 \mathrm{~h}$ after transfection and cell lysates were immunoblotted with the indicated antibodies to detect levels of mitochondria, Golgi and ER proteins

and the ability to both localize to the mitochondria and induce apoptosis $^{23}$ - functioned similar to wild-type PUMA in the induction of mitochondrial clustering, mitochondrial loss and LC3 puncta formation (Figure $3 \mathrm{~b}$ and data not shown).

If the induction of autophagy by PUMA is due to release of Beclin-1 from Bcl2/Bcl-xL, then it would not be predicted to be influenced by Bax or Bak expression. However, we have shown earlier that induction of apoptosis by PUMA is absolutely dependent on the presence of Bax or Bak. ${ }^{25}$ Surprisingly, the expression of PUMA in Bax/Bak double-null cells had no effect at all on mitochondrial morphology or maintenance (Figure 3c). PUMA protein expressed in these 
a
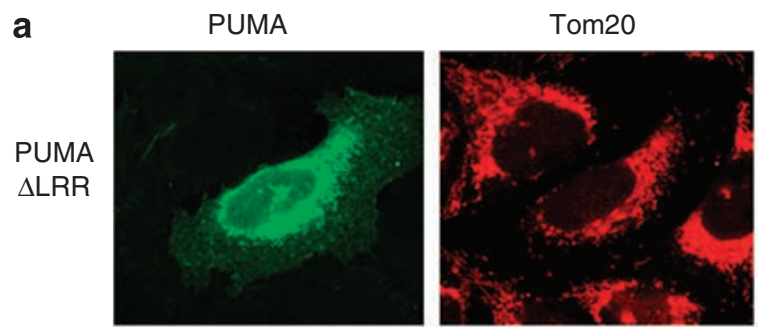

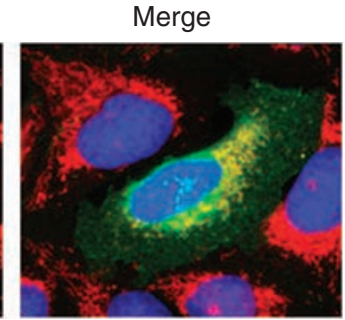

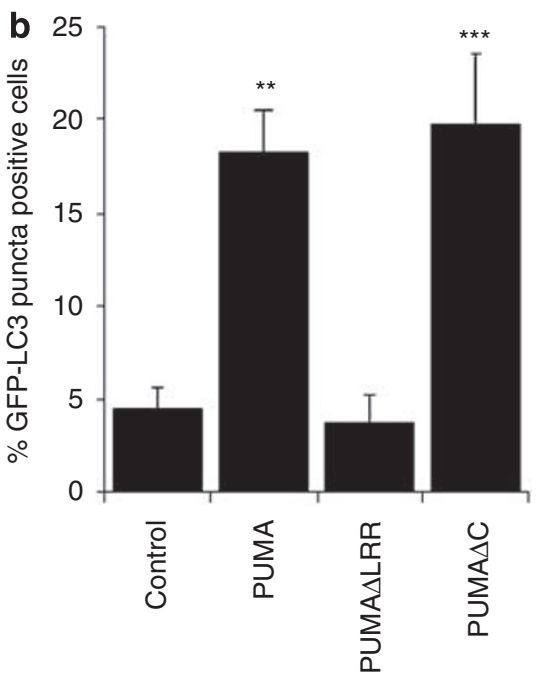

C
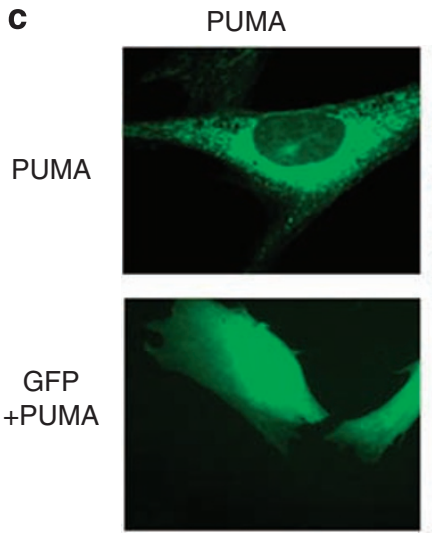

GFP
Tom20
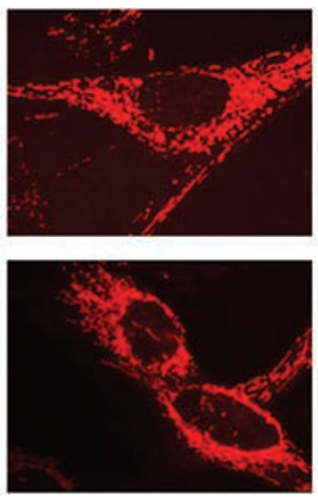
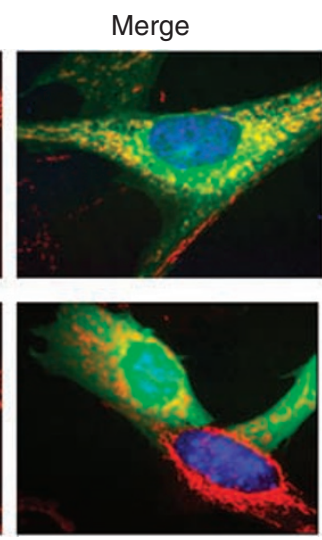

Figure 3 The ability of PUMA to affect mitochondria and LC3-punctae depends on Bax/Bak. (a) U2OS cells were transfected with PUMA $\triangle$ LRR-expression plasmid and subjected to immunofluorescence analysis $24 \mathrm{~h}$ later. PUMA $\triangle \mathrm{LRR}$ was detected using anti-FLAG antibody, whereas mitochondria were stained with anti-Tom20 antibody. (b) Quantitation of GFP-LC3 puncta formation. U2OS cells were infected with GFP-LC3-expressing adenovirus and then transfected with expression plasmids for the indicated proteins. Cells were maintained in the presence of $z$-VAD-fmk throughout the experiment. After $24 \mathrm{~h}$, cells were fixed and observed under a fluorescence microscope. Results are presented as mean percentage of GFP-LC3-positive cells showing puncta. Data are mean and S.D. of three independent experiments. ( ${ }^{* \star} P<0.001$, ${ }^{* \star *} P<0.005$ compared with control transfected cells). (c) Bax/Bak DKO MEFs were transfected with plasmids expressing the indicated proteins and subjected to immunofluorescence analysis after $24 \mathrm{~h}$. In the upper panel, PUMA was detected using an anti-Flag antibody, whereas mitochondria were stained using anti-Tom20 antibody. In the lower panel, GFP positivity indicated cells that co-expressed PUMA and showed maintenance of mitochondria (detected with anti-Tom20)

cells colocalized with Tom20 staining in a broadly spread reticulate network (upper panel), and GFP/PUMA co-transfected cells retained their mitochondria (lower panel).

The requirement of Bax/Bak for PUMA to induce the mitochondrial changes led us to test whether expression of Bax alone would be able to mediate similar effects. Subsequent experiments showed that expression of Bax could induce the mitochondrial clustering seen following
PUMA expression (Figure 4a). As expected, Bax also induced these mitochondrial changes in the Bax/Bak-null cells (data not shown). It should be noted that in these experiments transfection of Bax leads to the expression of activated Bax that localizes to the mitochondria and is competent to induce apoptosis. As seen following PUMA expression, perinuclear clustering of mitochondria in response to Bax occurred in the presence or absence of z-VAD-fmk (Supplementary Figure 

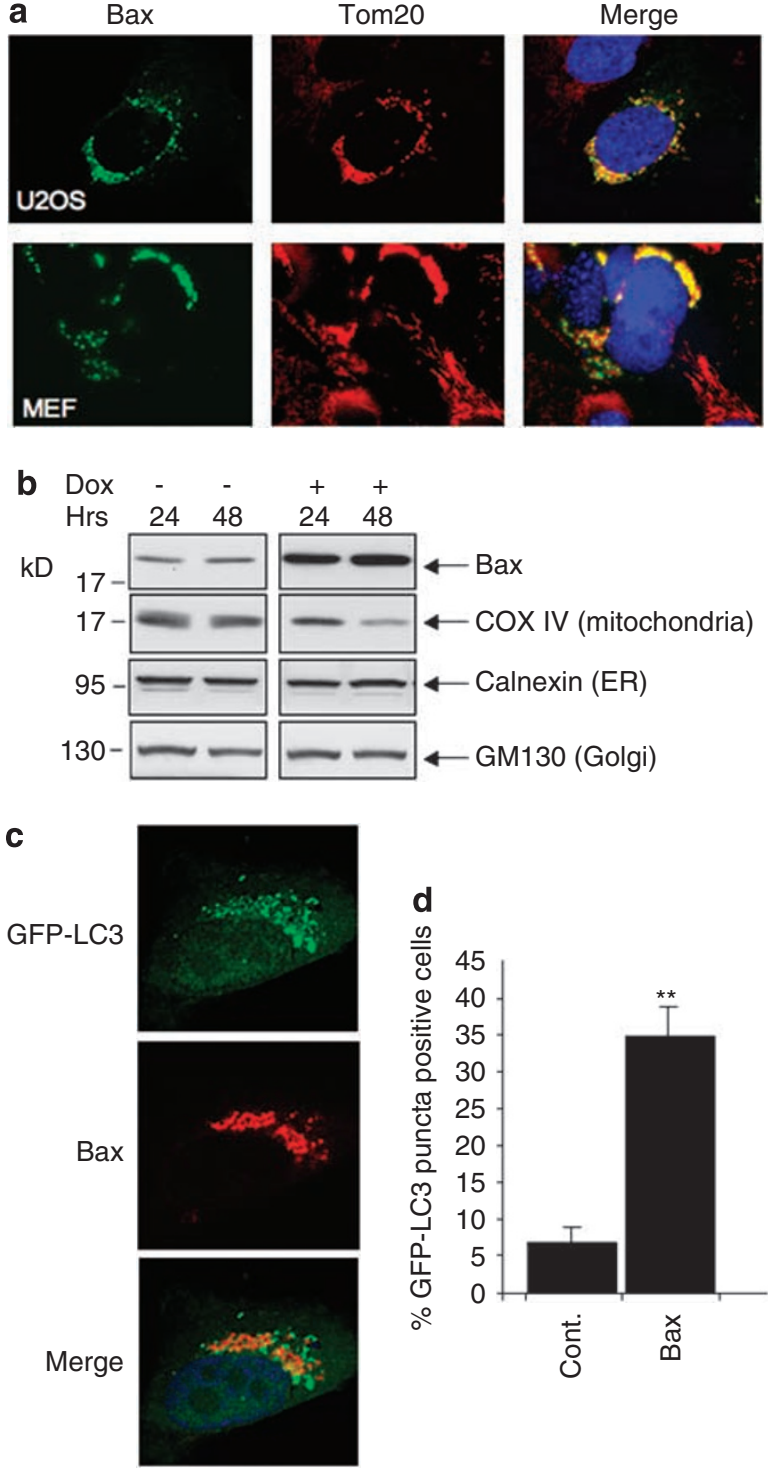

Figure 4 Bax functions similarly to PUMA to induce mitochondrial loss and accumulation of LC3 punctae. (a) Bax induces mitochondrial perinuclear accumulation and loss. U2OS and WT MEFs were transfected with Bax-expression plasmid and subjected to immunofluorescence analysis after $24 \mathrm{~h}$. Cells were maintained in the presence of z-VAD-fmk throughout the experiment. Bax was detected using anti-Bax antibody, whereas mitochondria were stained using antiTom20 antibody. (b) Saos-2 TetOn Bax cells were treated with doxycycline to induce Bax expression and maintained in the presence of z-VAD-fmk throughout the experiment. Cell were harvested 24 or $48 \mathrm{~h}$ after doxycycline treatment and cell lysates were immunoblotted with the indicated antibodies to detect the levels of mitochondria, Golgi and ER proteins. (c) U2OS cells were infected with GFP-LC3expressing adenovirus and then transfected with Bax-expression plasmid. Cells were maintained in the presence of z-VAD-fmk throughout the experiment. After $24 \mathrm{~h}$, cells were fixed, stained for Bax and observed under a fluorescence microscope. (d) Quantitation of GFP-LC3 puncta. Saos-2 TetOn Bax cells were infected with GFP-LC3-expressing adenovirus and then treated with doxycycline for $24 \mathrm{~h}$. Cells were maintained in the presence of z-VAD-fmk throughout the experiment. Cells were fixed and analyzed using a fluorescence microscope. Results are presented as mean percentage of GFP-LC3-positive cells showing puncta. Data are mean and S.D. of three independent experiments ${ }^{* *} P<0.001$ compared with control cells)
1C), and the loss of mitochondrial markers caused by the induction of Bax was not accompanied by a clear decrease in ER or golgi markers (Figure $4 b$ ). The ability of Bax to induce mitochondrial clustering and loss suggested that Bax may also be inducing autophagy. This was confirmed by showing that, similar to PUMA, expression of Bax induces LC3 puncta formation (Figure $4 \mathrm{c}$ and $\mathrm{d}$ and (Supplementary Figure $2 \mathrm{~B}$ )).

To further establish the effect of PUMA and Bax on autophagy, we examined the conversion from LC3-I to LC3-II by western blot, where LC3-II is detected as a faster migrating band. In agreement with the immunofluorescence studies, both PUMA and Bax expression enhanced the conversion of LC3, but this was not seen following expression of a $\mathrm{BH} 3$ domain mutant of PUMA (Figure 5a). Analysis by electron microscopy also showed the presence of vesicles consistent with the formation of autophagosomes following PUMA or Bax induction (Supplementary Figure 2C).

The accumulation of LC3 punctae could result from either an enhanced rate of autophagy, or a block in the later stages of autophagy to prevent degradation of the autophagosomes. We therefore examined the effect of chloroquine (which inhibits the lysosome/autophagosome fusion) on the levels of LC3-II. As expected, if PUMA is contributing to the enhancement of autophagy, the LC3-II levels were higher in PUMA/ chloroquine cells compared with chloroquine-only cell (Figure 5b). Taken together, our results suggest that PUMA enhances autophagy, that this effect requires Bax and/or Bak and that the autophagy induced by PUMA shows some selectivity in the removal of mitochondria. General autophagy can be assessed by an increased degradation of long-lived proteins, an effect that we observed in nutrient-starved cells, as expected (Figure 5c). Interestingly, however, we did not detect a change in the degradation of long-lived proteins following activation of PUMA, despite a clear loss of the mitochondrial marker COX IV (Figure 5c). These results support the suggestion that PUMA can lead to the specific loss of mitochondria, through mitophagy, without inducing a general and nonspecific autophagic response. It is also interesting to note that we did not see non-apoptotic cell death following PUMA or Bax induction in the presence of $z$ VAD-fmk after 3 days treatment (as assessed by $\mathrm{PI}$ exclusion). This is in contrast to cells undergoing general autophagy in response to ER stress, ${ }^{26}$ where necrotic death is detected. This may therefore also reflect the difference between general autophagy and the more selective mitophagy seen here.

Having found that Bax and PUMA can induce autophagy and the removal of mitochondria, we were interested in understanding whether this would have any effect on their apoptotic activity. The removal of mitochondria through mitophagy has been reported earlier and is thought to be triggered by mitochondrial membrane permeability transition caused by mitochondrial damage. ${ }^{27}$ Such a response would be entirely consistent with our observations that the autophagic effects of Bax and PUMA expression correlate with the ability to activate apoptosis. Most studies have indicated that the mitophagic response to mitochondrial damage is a prosurvival mechanism, removing damaged mitochondria and their associated apoptogenic signals. We therefore examined the contribution of autophagy to the sensitivity of 
a

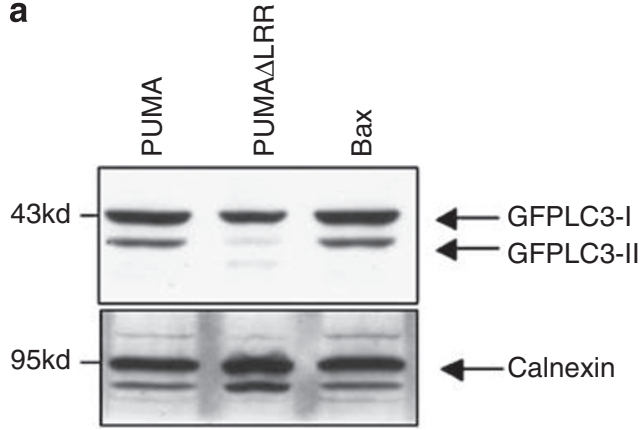

b
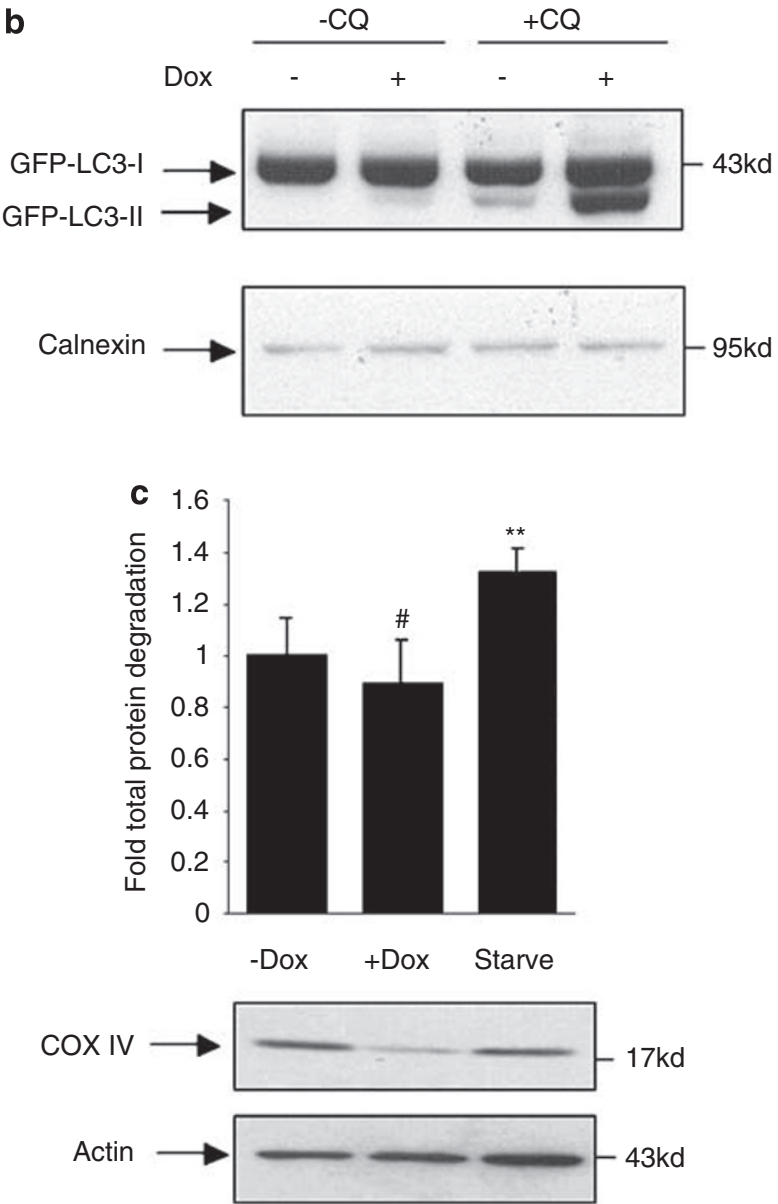

Figure 5 PUMA promotes autophagosome formation and loss of mitochondrial markers, but not general degradation of long-lived proteins. (a) HeLa cells stably expressing GFP-LC3 were transfected with plasmids expressing the indicated proteins, maintained in the presence of $z-V A D$-fmk and harvested after $24 \mathrm{~h}$. Cell lysates were then subjected to western blotting using anti-GFP antibodies to detect GFP-LC3-I and the faster migrating GFP-LC3-II. Calnexin was used as a loading control. (b) Inhibition of autophagosome degradation enhances levels of PUMAinduced GFP-LC3-II. Saos2 TetOn PUMA cells were infected with GFP-LC3expressing adenovirus and then treated with doxycycline for $24 \mathrm{~h}$. Chloroquine $(30 \mu \mathrm{M})$ was then added and cells were harvested after $9 \mathrm{~h}$. Cells were maintained in the presence of z-VAD-fmk throughout the experiment. Cell lysates were subjected to western blotting using anti-GFP antibodies to detect GFP-LC3-I and the faster migrating GFP-LC3-II. Calnexin was used as a loading control. (c) Protein stability was assessed in Saos2 TetOn PUMA cells following doxycycline treatment to induce PUMA or starvation ( ${ }^{*}$ o significant difference; ${ }^{* *} P$-value $<0.001$ compared with untreated cells). Western blot for COXIV protein levels in these samples is shown below. Actin was used as a loading control cells to Bax-induced apoptosis by reducing the expression of components of the autophagic pathway. Small interfering RNA (siRNA) targeted to Atg5 lowered the expression of the Atg 5 protein and partially rescued the mitochondrial loss induced by Bax expression (Figure 6a). Surprisingly, knockdown of Atg5 also significantly reduced Bax-induced apoptosis (Figure 6b), suggesting that the autophagic response contributes to the apoptotic response. However, we were concerned that this result may be complicated by the recent description of a direct role for Atg5 in apoptosis following cleavage by calpain ${ }^{22}$ - although we were unable to detect any increase in calpain activity following activation of either PUMA or Bax (data not shown). We therefore used siRNA to reduce the expression of other proteins required for autophagy and found that reduction of the expression of Atg7 or Atg10 also lowered the apoptotic response to Bax (Figure 6b). PUMA-induced apoptosis was also inhibited by the reduction of autophagic proteins Atg5, Atg7 or Atg10 (Figure 6c).

To try and elucidate the stage of apoptosis that was affected by reducing the expression of Atg5, we carried out cell fractionation to evaluate the effect on cytochrome $c$ release. As expected, the expression of Bax induced the release of cytochrome $c$ from the mitochondria (Figure 7a), a response that was inhibited by the reduction of Atg5 expression. Our studies suggest that, rather unexpectedly, autophagy may be contributing to the efficient release of cytochrome $c$ and so the induction of apoptosis. However, it is also likely that mitochondrial membrane permeabilization induced by Bax is a trigger to induce autophagy - particularly the selective removal of these damaged mitochondria. We therefore tried to determine whether the formation of autophagosomes occurred before or after cytochrome $c$ release. However, despite several attempts in different systems we were unable to clearly dissociate the appearance of LC3 puncta from the release of cytochrome $c$ following either Bax or PUMA expression (Supplementary Figure 3).

Another feature frequently associated with apoptosis is the loss of mitochondrial inner membrane potential. Using the tetramethylrhodamine ethyl ester (TMRE) dye, which binds to mitochondria in a membrane potential-dependent manner, we confirmed that expression of Bax resulted in the appearance of a population of cells with decreased potential. Interestingly, this population of cells was reduced following siRNA depeletion of Atg5 (Figure 7b), suggesting that mitophagy contributes to the Bax-induced apopotosis by enhancing both cytochrome $c$ release and loss of mitochondrial membrane potential.

\section{Discussion}

We have shown that the BH3-only protein PUMA can induce autophagy concurrently with apoptosis, and that this appears to result in the selective removal of mitochondria. The induction of autophagy by PUMA requires its $\mathrm{BH} 3$ domain and is dependent on the presence of Bax or Bak. Bax alone can also induce mitochondrial-selective autophagy in the absence of PUMA activation, and we find a close correlation between the induction of apoptosis and autophagy in these systems. 

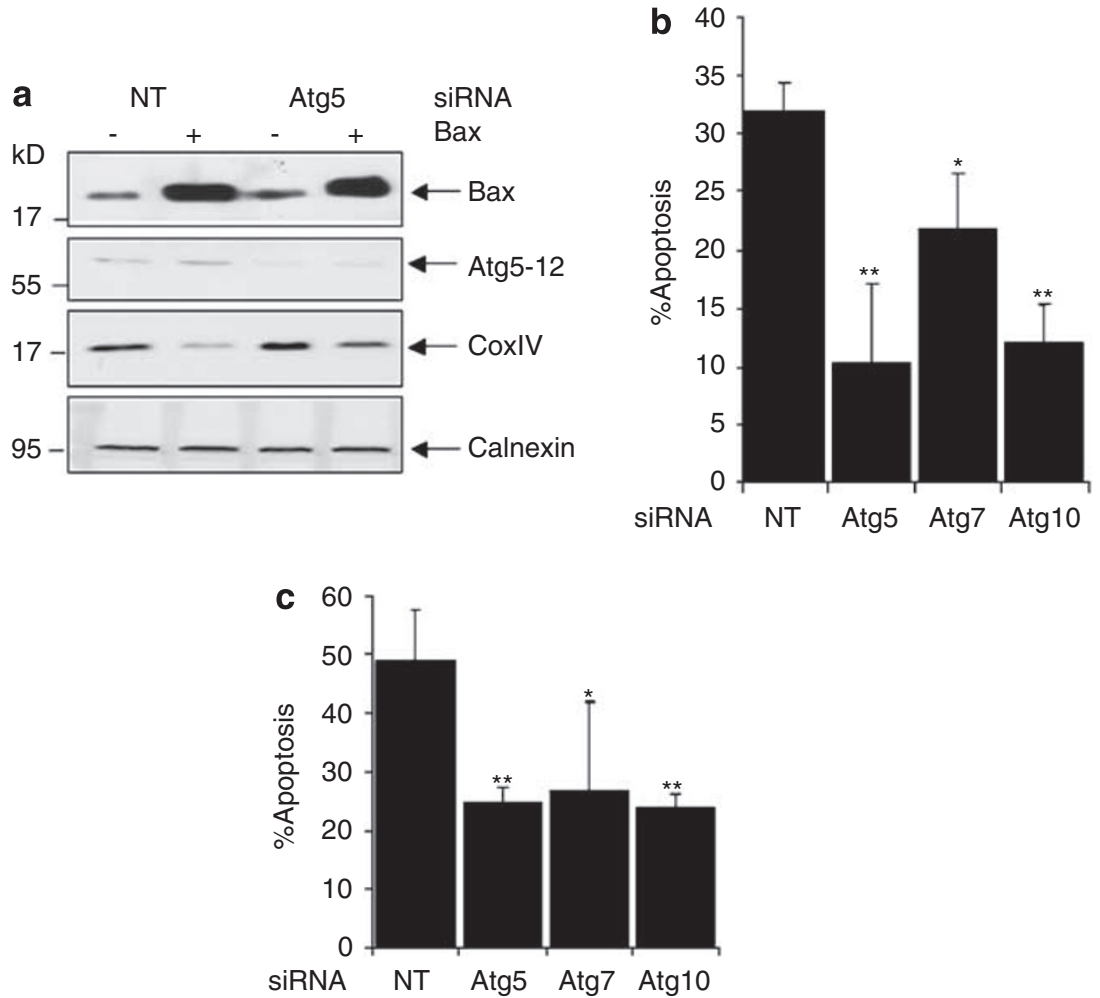

Figure 6 Inhibition of autophagy partially rescues the mitochondria loss and apoptosis induced by Bax and PUMA. (a) Saos-2 TetOn Bax cells were transfected with nontargeting siRNA or siRNA against Atg5. After $72 \mathrm{~h}$, doxycycline was added to induce Bax expression in the presence of z-VAD-fmk. After $24 \mathrm{~h}$ induction, cells were harvested for western blot analysis. Cell lysates were immunoblotted and probed with antibodies against the indicated proteins. Anti-Atg12 was used to detect Atg5-12 complex to confirm the decrease in Atg5 expression. Anti-COXIV antibody was used to detect mitochondria protein levels. (b) Saos-2 TetOn Bax cells were transfected with non-targeting siRNA as well as siRNA against Atg5, Atg7 and Atg10. After $72 \mathrm{~h}$, doxycycline was added to induce Bax expression. After $24 \mathrm{~h}$, cells were harvested and apoptosis was evaluated by flow cytometry. Results are the mean and S.D. for at least three independent experiments. ${ }^{*} P$-value $<0.05$, ${ }^{\star *} P$-value $<0.001$ compared with non-targeted siRNA transfected cells). (c) Saos-2 TetOn PUMA cells were transfected with non-targeted siRNA or siRNA against Atg5, Atg7 and Atg 10. After 72 h, doxycycline was added to induce PUMA expression. After $24 \mathrm{~h}$, cells were harvested and apoptosis was evaluated by flow cytometry. Results are the mean and S.D. for at least three independent experiments ( ${ }^{*} P$-value $<0.05,{ }^{* *} P$-value $<0.001$ compared with non-targeted siRNA transfected cells)

A growing body of evidence supports a distinction between general autophagy and the specific removal of mitochondria through autophagic mechanisms, also termed mitophagy. ${ }^{28}$ General or non-selective autophagy is an important response to maintain metabolism and allow cells to survive limited periods of hypoxia, starvation or nutrient deprivation. This is a short-term survival solution, however, and extended or overactivated autophagy can result in the death of cells following extensive self-digestion of organelles and cytoplasm. This autophagic response is dependent on Beclin-1 and can be triggered by BH3-only proteins, such as BNIP3 and Bad, which disrupt the inhibitory interaction between $\mathrm{Bcl} 2 / \mathrm{Bcl}-\mathrm{xL}$ and Beclin-1. More selective forms of autophagy that result in the engulfment and degradation of only mitochondria have been suggested to play an important role in the removal of damaged mitochondria, although the mechanism underlying the selectivity are not clear. Our results suggest that PUMA functions through Bax to induce mitochondrial selective autophagy that is triggered simultaneously with cytochrome $c$ release. It would appear that induction of autophagy (as assessed by autophagosome appearance) does not induce or precede the release of cytochrome $c$, but rather the same stimulus (such as changes in mitochondrial membrane potential) concurrently induces both these events.

The autophagic signal induced by PUMA is clearly different from that activated by starvation or ER stress, which are independent of Bax/Bak. ${ }^{12,26}$ The dependence of PUMAinduced autophagy on Bax/Bak indicates that PUMA does not function directly to release Beclin-1 from $\mathrm{Bcl} 2 / \mathrm{Bcl}-\mathrm{xL}-$ at least in these model systems. This is further supported by the observation that Bax - which does not bind Beclin-1 and is unable to disrupt the interaction between Bcl2/XI and Beclin$1^{29}$ - functions to induce mitochondrial autophagy in a manner very similar to PUMA. This activity of Bax would seem to be linked with the ability to induce apoptosis, as a recent study has shown that expression of cytoplasmic Bax (which is inactive) cannot induce autophagy in Bax/Bak-null cells. ${ }^{26}$ Interestingly, we also found that knockdown of Beclin1 expression by siRNA did not prevent apoptosis in response to PUMA or Bax, even though reduction of Beclin-1 expression was able to inhibit the formation of autophagosomes in response to starvation (data not shown). It therefore seems possible that PUMA/Bax-induced autophagy is Atg5-, Atg7and Atg10-dependent but Beclin-1-independent, a situation very similar to that described recently for neuronal autophagy 
a
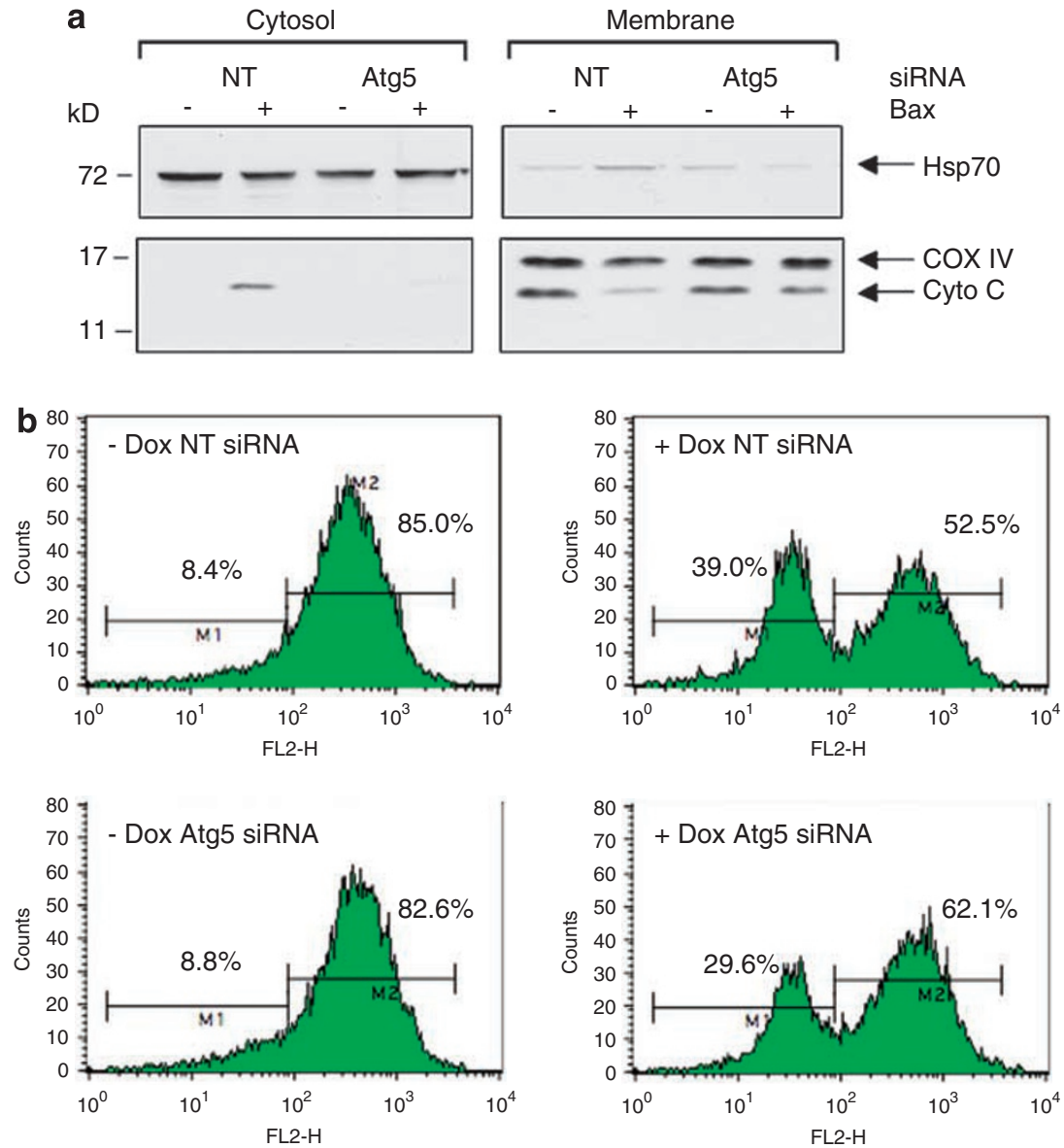

Figure 7 Inhibition of autophagy inhibits cytochrome $c$ release and decrease of mitochondrial membrane potential induced by Bax. Saos-2 TetOn Bax cells were transfected with non-targeting siRNA or siRNA against Atg5. After $72 \mathrm{~h}$, doxycycline was added to induce Bax expression in the presence of z-VAD-fmk. (a) After $24 \mathrm{~h}$ Bax induction, cells were harvested and subjected to subcellular fractionation. Equivalent amounts of each fraction were subjected to western blot analysis to detect cytochrome $c$ levels and marker proteins; COXIV for membrane fraction and Hsp70 for cytosolic fraction. (b) After $24 \mathrm{~h}$ Bax induction, cells were stained with TMRE to determine alterations in mitochondrial membrane potential $\left(\Delta \psi_{\mathrm{m}}\right)$. A decrease in $\Delta \psi_{\mathrm{m}}$ is shown by the appearance of a peak with lower fluorescence intensity. The experiment was carried out at least three times and gave similar results

induced by the parkinsonian neurotoxin 1-methyl-4-phenylpyridinium $(\operatorname{MPP}(+)) \cdot{ }^{30}$ Similar to PUMA and $\operatorname{Bax}, \operatorname{MPP}(+)$ induces mitochondrial injury, autophagy and mitochondrial degradation that is inhibited by siRNA knockdown of Atg5, Atg7 and Atg8 but occurs independently of Beclin-1.

It seems likely that alterations in mitochondrial membrane potential-induced Bax/Bak can signal to both apoptosis and mitochondrial autophagy. Indeed, earlier studies have shown that mitochondria that have sustained stress-induced damage, such as MPT or MOMP, can be removed by selective mitochondrial autophagy. ${ }^{13,27,30,31}$ Furthermore, in yeast mitochondrial dysfunction (as measured by impaired mitochondrial membrane potential) also induces mitophagy, which is followed by cell death. ${ }^{32}$ What the precise signal from the altered mitochondrial membranes to autophagy is remains to be determined. Intriguingly, some $\mathrm{BH}$-only proteins, which would be expected to activate Bax and induce the pathways described here - such as BNIP3 - also induce mitophagy through the activation of Beclin-1. ${ }^{14}$ It remains possible that in different cell systems, or in response to different signals, PUMA may also engage this pathway.
The role of PUMA in p53-induced autophagy remains to be resolved. p53 transcriptionally activates the expression of DRAM ${ }^{33}$ which can mediate the autophagic response. In cells where p53 drives DRAM expression, we were unable to identify an essential role for PUMA in the activation of autophagy (data not shown), consistent with DRAM being sufficient for this response. However, the response to p53 is complicated, with evidence that p53 can also function to repress autophagy. ${ }^{34}$ The ultimate response to p53 activation and the contribution of various p53 activities is therefore likely to be strongly stimulus and cell-type dependent.

Despite descriptions of autophagic cell death, there is a consensus that autophagy itself is more usually a survival mechanism, and although dying cells can show characteristics of autophagy, this is generally not the mechanism through which the cells die. ${ }^{35}$ However, it is possible that autophagy can contribute to the induction of other death responses, such as apoptosis. In light of the coordinate activation of both autophagic and apoptotic signals by PUMA and Bax, we examined whether autophagy could impact the apoptotic response. The impact of autophagy on 
apoptosis is highly context-dependent, with compelling evidence that autophagy can both inhibit or promote apoptosis, depending on the system. As discussed above, general autophagy in response to starvation clearly plays a survival role, although a number of studies have also shown that general autophagy can play a role in promoting or initiating apoptosis. ${ }^{17,19}$ There are many examples of systems in which a reduction or inhibition of autophagy leads to a reduction in apoptosis or cell death. ${ }^{18,30,31,33,36-38}$ The role for mitochondrial-specific autophagy in the regulation of apoptosis is similarly complex. Although selective removal of damaged mitochondria by autophagy has been proposed to protect or reduce the apoptotic signal, ${ }^{39}$ there are situations where mitophagy appears to contribute to apoptosis. Most interestingly, inhibition of the mitophagy associated with $\operatorname{MPP}(+)$-induced mitochondrial damage confers protection from $\operatorname{MPP}(+)$-induced cell death in a manner reminiscent of the results described here with PUMA and Bax. ${ }^{30,38}$ Bax has also been shown to induce both mitophagy and general autophagy, as well as death, in yeast. Interestingly, loss of expression of Uth1P - a protein that is specifically required for mitophagy - delays the rapid and regulated cell death induced by Bax, although these cells then die through necrosis. ${ }^{40}$

Taken together, our data support a role for PUMA or Baxinduced mitochondrial autophagy in the promotion of apoptosis. Importantly, however, while this mitophagy can enhance apoptosis, it does not appear to induce cell death by itself in the short term, although loss of mitochondria is most likely to be detrimental to the cell in the long term. The mechanism for this proapoptotic activity of mitochondrial autophagy remains unclear. It is possible that the process of autophagy enhances the breakdown of the mitochondria and so facilitates the release of cytochrome $c$ and/or SMAC/DIABLO. Alternatively, the removal of mitochondria might promote cell death by reducing the capacity of the cell for energy production and survival. $^{28}$

\section{Materials and Methods}

Reagents and antibodies. All reagents used in this study were from Sigma unless otherwise indicated. For western blot and immunofluorescence experiments, cells were treated with $50 \mu \mathrm{M} \mathrm{z-VAD-fmk} \mathrm{(Bachem)} \mathrm{to} \mathrm{inhibit} \mathrm{caspase} \mathrm{activity} \mathrm{and}$ cell death.

The following antibodies were used Flag M2 (F3165), Sigma; calnexin H-70 (sc-11397), Tom20 FL-145 (sc-11415) and Bax clone 2D2 (sc-20067) from Santa Cruz Biotechnology; OxPhos Complex IV subunit IV 10G8 (COXIV) (A21347) and OxPhos Complex IV subunit II 12C4 (COXII) (A6404) from Molecular Probes; cytochrome $c$ (556433) and GM 130 (610832) from BD Biosciences; HSP 70 from Transduction Labs; Apg12L (AP1816a) from Abgent and GFP (7.1/13.1) from Roche.

Cell lines and plasmids. U2OS (osteosarcoma), hTERT-RPE (telomeraseimmortalized human retinal pigment epithelial cells, Clontech), WT MEF (wild-type mouse embryonic fibroblast) and Bax-I- Bak-I- double knockout (DKO) MEF (kind gift of Craig Thompson, Abramson Family Cancer Research Institute, University of Pennsylvania, Philadelphia), Saos-2 TetOn PUMA-inducible ${ }^{41}$ and Saos-2 TetOn Bax-inducible cell lines ${ }^{42}$ have been described earlier. HeLa cell line stably expressing GFP-LC3 was generated by hygromycin selection of cells infected with pBabe-Hygro retrovirus expressing BamHI-EcoRI subcloned EGFP-LC3. ${ }^{24}$ All cells were maintained in Dulbecco's modified Eagle's medium (DMEM) supplemented with $10 \%$ fetal bovine serum, $2 \mathrm{mM}$ glutamine and antibiotics. Flag-tagged PUMA, PUMA $\triangle$ LRR, PUMA $\triangle \mathrm{C}$ (N164) and D01-tagged Bax have been described earlier. ${ }^{23,42}$ GFP-PUMA and DSRed-PUMA were constructed by cloning PUMA into pEGFP-N1 (Clontech) and DSRed-N1 vectors, respectively.
DNA and siRNA transfections. Cells were transfected with indicated plasmids (generally $4 \mu \mathrm{g}$ per $60 \mathrm{~mm}$ dish) or induced with doxycycline $(0.5$ and $1 \mu \mathrm{g} /$ $\mathrm{ml}$ doxycycline for Saos-2 TetOn PUMA and Bax, respectively). DNA transfections were carried out using Lipofectamine 2000 (Invitrogen) according to the manufacturer's instructions. siRNA transfections were used to knock down protein levels of autophagic genes. Sequences of siRNA used are as follows ATG5 (CAUCUGAGCUACCCGGAUAUU); ${ }^{33}$ ATG7 (GGAGUCACAGCUCUUCCUU); ${ }^{30}$ ATG10 (GGAGUUCAUGAGUGCUAUA). ${ }^{43}$ Nontargeted siRNA purchased from Dharmacon was used as control. siRNA transfections were carried out using HiPerfect reagent (Qiagen) according to the manufacturer's instructions. Cells were transfected at a concentration of $25 \mathrm{nM}$ siRNA twice on sequential days to enhance knock down. The cells were replated for various experiments $48 \mathrm{~h}$ after siRNA transfection, left overnight and then treated with doxycycline. Induced cells were harvested at indicated times for various assays.

Western blotting. For western blot analysis, transfected or induced cells were maintained in the presence of $50 \mu \mathrm{M} \mathrm{z}$-VAD-fmk and harvested and lysed in 1\% CHAPS buffer ( $150 \mathrm{mM} \mathrm{NaCl}, 10 \mathrm{mM}$ HEPES (pH 7.4), 1\% CHAPS) after the indicated time. The protein content of the clarified cell lysates were determined and equal amounts were subjected to SDS-PAGE and western blotting. For the chloroquine experiments, Saos2 TetOn PUMA cells were infected with adenovirus expressing GFP-LC3, left overnight and then treated with doxycycline to induce PUMA. $24 \mathrm{~h}$ later, $30 \mu \mathrm{M}$ chloroquine was added to the cells and left for $9 \mathrm{~h}$. Cells were then harvested and processed for western blotting as described above. Calnexin protein levels were detected to confirm equal loading of protein content.

Subcellular fractionation. Saos-2 TetOn cells in $100 \mathrm{~mm}$ tissue culture dishes were transfected with the indicated siRNA, treated with doxycycline and then subjected to subcellular fractionation using the ProteoExtract Subcellular Proteome Extraction kit (Calbiochem) according to the manufacturer's instructions. Equal amounts of membrane/organelle and cytosol fraction was separated using SDSPAGE followed by western blotting to detect cytochrome $c$ release and various marker proteins; Hsp70 for cytosol fractions and COXIV for membrane/organelle fractions.

Immunofluorescence. Cells were plated on sterile glass coverslips (in 12well tissue culture dishes) and transfected with $1 \mu \mathrm{g}$ of the indicated plasmids or treated with doxycycline to induce PUMA or Bax. All experiments were carried out in the presence of $z-V A D$-fmk unless otherwise indicated. After $24 \mathrm{~h}$, the cells were washed with PBS and then fixed in ice-cold $4 \%$ paraformaldehyde in PBS for 10 min at room temperature. After fixation, the cells were permeabilized in PBS containing $0.2 \%$ Triton-X100 for $5 \mathrm{~min}$. The cells were blocked in PBS containing $0.5 \%$ bovine serum albumin (blocking solution) at room temperature for $30 \mathrm{~min}$. and then incubated overnight at $4^{\circ} \mathrm{C}$ with a combination of the indicated antibodies in blocking solution. The cells were washed three times with PBS and incubated for $1 \mathrm{~h}$ at room temperature with a mixture of donkey anti-mouse FITC-conjugated antibody and donkey anti-rabbit Cy3-conjugated antibody (both from Jackson ImmunoResearch Laboratories) in blocking solution containing $1 \mu \mathrm{g}$ of DAPI (4',6 -diamidino-2phenylindole) (Sigma) per $\mathrm{ml}$. The cells were washed three times with PBS, and slides were mounted with Hard-Set Vectashield (Vector). Cells were observed and images obtained with either a Zeiss Axiovert fluorescent microscope or a Bio-Rad Radiance 2000 confocal microscope. In the merged images, DAPI staining was also shown to indicate the nucleus.

Apoptosis assay. Saos-2 TetOn cells were transfected with the indicated siRNAs and then treated with doxycycline to induce PUMA or Bax expression. Cells were harvested $24 \mathrm{~h}$ later and analyzed by flow cytometry using a FACSCalibur flow cytometer (488-nm argon laser excitation source) (BD Biosciences). Apoptosis was assessed by evaluating caspase activation using the CaspACE FITC-VAD-fmk in situ marker from Promega.

Quantitation of GFP-LC3 puncta. Saos-2 TetOn cells were infected with adenovirus expressing GFP-LC3 and then treated with doxycycline to induce PUMA or Bax expression. At the indicated times, cells were fixed with 4\% PFA and then observed under a fluorescence microscope. Cells were classified as having a predominantly diffuse GFP stain or having numerous punctate structures representing autophagosomes. At least 100 cells were scored in each of three or more independent experiments. In experiments comparing the appearance of GFPLC3 puncta to release of cytochrome $c$, cells were subjected to immunofluorescence staining with cytochrome $c$ antibody before being evaluated under the microscope. 
Measurement of mitochondrial membrane potential. Tetramethylrhodamine ethyl ester (Molecular Probes) was used to evaluate changes in mitochondria membrane potential. Doxycycline-treated cells were harvested and resuspended in PBS containing $50 \mathrm{nM}$ TMRE. After incubation at $37^{\circ} \mathrm{C}$ for $20 \mathrm{~min}$ samples were analyzed by flow cytometry. Cells with decreased mitochondrial membrane potential exhibited lower TMRE uptake (peak with lower fluorescence intensity).

Statistical analysis. Statistical significance of experiments was determined using Student's $t$-test, with a significance level of $P<0.05$.

Calpain activation assay. Calpain activity was measured using the CalpainGlo kit (Promega). Saos2 TetOn cells were induced with doxycycline for $24 \mathrm{~h}$. Cells were then harvested and resuspended in hepes lysis buffer (10 mM HEPES pH 7.5 $10 \mathrm{mM}$ DTT, $1 \mathrm{mM}$ EDTA and $1 \mathrm{mM}$ EGTA). Cells were then centrifuged at full speed and the supernatant was used for the assay (according to the manufacturer's instructions) as well as protein quantification.

Electron microscopy. Saos2 TetOn PUMA and Bax cells were plated in $100 \mathrm{~mm}$ tissue culture dishes at near confluency and induced with doxycycline. After $24 \mathrm{~h}$, media was replaced with fixative (4\% paraformaldehyde $/ 2.5 \%$ glutaraldehyde in $0.2 \mathrm{M}$ PIPES) and the cells were fixed for $1 \mathrm{~h}$. The fixative was then removed and the cells were scraped into $1 \mathrm{ml}$ of fixative, transferred to a microfuge tube and spun down. Pellets were treated with 1\% osmium tetroxide (Agar Scientific), dehydrated in ethanol and propylene oxide before embedding in Durcupan resin (Sigma). Sections $(60-70 \mathrm{~nm})$ were cut on a Leica Ultracut UCT ultramicrotome and mounted on hexagonal, 100-mesh copper grids. Sections were stained with uranyl acetate and lead citrate and examined in a Jeol 1200 EX electron microscope. Images were recorded on imaging plates and processed in a Ditabis plate scanner.

Measurement of degradation of long-lived proteins. Cells were labeled for $8 \mathrm{~h}$ with L-[35S]Met/Cys $(5 \mu \mathrm{Ci} / \mathrm{ml})$ (Amersham), washed three times in PBS, then incubated overnight in serum-free DMEM with $2 \mathrm{mM}$ unlabeled L-Met/ Cys (plus z-VAD-fmk, plus/minus doxycycline). Subsequently, cells were washed and incubated in fresh DMEM (plus z-VAD-fmk plus/minus doxycycline) or EBSS plus z-VAD-fmk for $8 \mathrm{~h}$, whereupon the levels of degraded protein were calculated as described earlier. ${ }^{43}$

Acknowledgements. We thank Dr. Aviva Tolkovsky (University of Cambridge) for the kind gift of GFP-LC3-expressing adenovirus. This work was funded by Cancer Research UK and the West of Scotland Women's Bowling Association.

1. Hanahan D, Weinberg RA. The hallmarks of cancer. Cell 2000; 100: 57-70.

2. Green DR, Kroemer G. The pathophysiology of mitochondrial cell death. Science 2004 305: 626-629.

3. Cory S, Adams JM. The Bcl2 family: regulators of the cellular life-or-death switch. Nat Rev Cancer 2002; 2: 647-656.

4. Wei MC, Zong WX, Cheng EH, Lindsten T, Panoutsakopoulou V, Ross AJ et al. Proapoptotic BAX and BAK: a requisite gateway to mitochondrial dysfunction and death Science 2001; 292: 624-626.

5. Chipuk JE, Green DR. How do BCL-2 proteins induce mitochondrial outer membrane permeabilization? Trends Cell Biol 2008; 18: 157-164

6. Yu J, Zhang L. No PUMA, no death: implications for p53-dependent apoptosis. Cancer Cell 2003; 4: 248-249.

7. Halazonetis TD, Gorgoulis VG, Bartek J. An oncogene-induced DNA damage model for cancer development. Science 2008; 319: 1352-1355.

8. Oren M. Decision making by p53: life, death and cancer. Cell Death Differ 2003; 10 : 431-442.

9. Mizushima N. Autophagy: process and function. Genes Dev 2007; 21: 2861-2873.

10. Kim I, Rodriguez-Enriquez S, Lemasters JJ. Selective degradation of mitochondria by mitophagy. Arch Biochem Biophys 2007; 462: 245-253.

11. Levine B, Kroemer G. Autophagy in the pathogenesis of disease. Cell 2008; 132 $27-42$

12. Lum JJ, Bauer $D E$, Kong $M$, Harris $M H$, Li $C$, Lindsten $T$ et al. Growth facto regulation of autophagy and cell survival in the absence of apoptosis. Cell 2005; 120: 237-248.

13. Colell A, Ricci JE, Tait S, Milasta S, Maurer U, Bouchier-Hayes L et al. GAPDH and autophagy preserve survival after apoptotic cytochrome $c$ release in the absence of caspase activation. Cell 2007; 129: 983-997.
14. Zhang H, Bosch-Marce M, Shimoda LA, Tan YS, Baek JH, Wesley JB et al. Mitochondrial autophagy is an HIF-1-dependent adaptive metabolic response to hypoxia. $\mathrm{J} \mathrm{Biol} \mathrm{Chem}$ 2008; 283: 10892-10903.

15. Mathew R, Karantza-Wadsworth V, White E. Role of autophagy in cancer. Nat Rev2007; 7: 961-967.

16. Maiuri MC, Tasdemir E, Criollo A, Morselli E, Vicencio JM, Carnuccio R et al Control of autophagy by oncogenes and tumor suppressor genes. Cell Death Differ 2009; 16: 87-93.

17. Scarlatti F, Granata R, Meijer AJ, Codogno P. Does autophagy have a license to kill mammalian cells? Cell Death Differ 2008; 16: 12-20.

18. Berry DL, Baehrecke EH. Growth arrest and autophagy are required for salivary gland cell degradation in Drosophila. Cell 2007; 131: 1137-1148.

19. Maiuri MC, Zalckvar E, Kimchi A, Kroemer G. Self-eating and self-killing: crosstalk between autophagy and apoptosis. Nat Rev Mol Cell Biol 2007; 8: 741-752.

20. Pattingre S, Tassa A, Qu X, Garuti R, Liang XH, Mizushima N et al. Bcl-2 antiapoptotic proteins inhibit Beclin 1-dependent autophagy. Cell 2005; 122: 927-939.

21. Maiuri MC, Le Toumelin G, Criollo A, Rain JC, Gautier F, Juin P et al. Functional and physical interaction between $\mathrm{Bcl}-\mathrm{X}(\mathrm{L})$ and a $\mathrm{BH} 3-$ like domain in Beclin-1. EMBO J 2007; 26: 2527-2539.

22. Yousefi S, Perozzo R, Schmid I, Ziemiecki A, Schaffner T, Scapozza L et al. Calpain-mediated cleavage of Atg5 switches autophagy to apoptosis. Nat Cell Biol 2006; 8 : 1124-1132.

23. Yee KS, Vousden KH. Contribution of membrane localization to the apoptotic activity of PUMA. Apoptosis 2008; 13: 87-95.

24. Kabeya Y, Mizushima N, Ueno T, Yamamoto A, Kirisako T, Noda T et al. LC3, a mammalian homologue of yeast Apg8p, is localized in autophagosome membranes after processing. EMBO J 2000; 19: 5720-5728.

25. Melino G, Bernassola F, Ranalli M, Yee K, Zong WX, Corazzari M et al. p73 induces apoptosis via PUMA transactivation and Bax mitochondrial translocation. J Biol Chem 2004; 279: 8076-8083.

26. Ullman E, Fan Y, Stawowczyk M, Chen HM, Yue Z, Zong WX. Autophagy promotes necrosis in apoptosis-deficient cells in response to ER stress. Cell Death Differ 2008; 15 : 422-425.

27. Elmore SP, Qian T, Grissom SF, Lemasters JJ. The mitochondrial permeability transition initiates autophagy in rat hepatocytes. FASEB J 2001; 15: 2286-2287.

28. Kundu M, Thompson CB. Macroautophagy versus mitochondrial autophagy: a question of fate? Cell Death Differ 2005; 12 (Suppl 2): 1484-1489.

29. Erlich S, Mizrachy L, Segev O, Lindenboim L, Zmira O, Adi-Harel S et al. Differential interactions between Beclin 1 and Bcl-2 family members. Autophagy 2007; 3: $561-568$

30. Zhu JH, Horbinski C, Guo F, Watkins S, Uchiyama Y, Chu CT. Regulation of autophagy by extracellular signal-regulated protein kinases during 1-methyl-4-phenylpyridinium-induced cell death. Am J Pathol 2007; 170: 75-86.

31. Xue L, Fletcher GC, Tolkovsky AM. Autophagy is activated by apoptotic signalling in sympathetic neurons: an alternative mechanism of death execution. Mol Cell Neurosci 1999; 14: 180-198.

32. Priault M, Salin B, Schaeffer J, Vallette FM, di Rago JP, Martinou JC. Impairing the bioenergetic status and the biogenesis of mitochondria triggers mitophagy in yeast. Cell Death Differ 2005; 12: 1613-1621

33. Crighton D, Wilkinson S, O'Prey J, Syed N, Harrison PR, Gasco M et al. DRAM, a p53-induced modulator of autophagy, is critical for apoptosis. Cell 2006; 14: 121-134.

34. Tasdemir E, Maiuri MC, Galluzzi L, Vitale I, Djavaheri-Mergny M, D'Amelio M et al. Regulation of autophagy by cytoplasmic p53. Nat Cell Biol 2008; 10: 676-687.

35. Kroemer G, Levine B. Autophagic cell death: the story of a misnomer. Nat Rev Mol Cell Biol 2008; 9: 1004-1010.

36. Espert L, Denizot M, Grimaldi M, Robert-Hebmann V, Gay B, Varbanov M et al. Autophagy is involved in T cell death after binding of HIV-1 envelope proteins to CXCR4. J Clin Invest 2006; 116: 2161-2172.

37. Kunchithapautham K, Rohrer B. Apoptosis and autophagy in photoreceptors exposed to oxidative stress. Autophagy 2007; 3: 433-441.

38. Chu CT, Zhu J, Dagda R. Beclin 1-independent pathway of damage-induced mitophagy and autophagic stress: implications for neurodegeneration and cell death. Autophagy 2007; 3: 663-666.

39. Meier $\mathrm{P}$, Vousden KH. Lucifer's labyrinth - ten years of path finding in cell death. Mol Cell 2007; 28: 746-754

40. Kissova I, Plamondon LT, Brisson L, Priault M, Renouf V, Schaeffer J et al. Evaluation of the roles of apoptosis, autophagy, and mitophagy in the loss of plating efficiency induced by Bax expression in yeast. J Biol Chem 2006; 281: 36187-36197.

41. Nakano K, Vousden KH. PUMA, a novel pro-apopototic gene, is induced by p53. $\mathrm{Mol} \mathrm{Cell}$ 2001; 7: 683-694.

42. Phillips AC, Bates S, Ryan KM, Helin K, Vousden KH. Induction of DNA synthesis and apoptosis are separable functions of E2F-1. Genes and Dev 1997; 11: 1853-1863.

43. Boya P, Gonzalez-Polo RA, Casares N, Perfettini JL, Dessen P, Larochette N et al. Inhibition of macroautophagy triggers apoptosis. Mol Cell Biol 2005; 25: 1025-1040. 